\title{
Vulvar pruritus in a postmenopausal woman
}

\section{Emily Caro-Bruce MD, Gabriel Flaxman MD}

Competing interests: None declared.

This article has been peer reviewed.

\section{Correspondence to:}

Emily Caro-Bruce, Gabriel Flaxman, gabriel.flaxman@kp.org

CMAJ 2014. DOI:10.1503 /cmaj.130665 emily.x.caro-bruce@kp.org;

A 62-year-old woman presents with chronic vulvar pruritus. She treated herself for yeast vaginitis without improvement. She has had progressive dyspareunia and intermittent difficulty with defecation, as well as insomnia, which she attributes to ongoing itch. On examination, white vulvar plaques are noticed with fissuring. Results of urine studies are normal, and a recent wet mount of a vaginal swab was negative for the $\mathrm{KOH}$ "whiff" test, clue cells, yeast and Trichomonas. Her last Papanicolaou smear a year ago was normal.

\section{What diagnoses should be considered?}

Lichen sclerosus of the vulva is a chronic dermatologic condition characterized by pruritus, inflammation and tissue scarring, most often affecting postmenopausal women. ${ }^{1-3}$ The chief symptom is vulvar itching, which sometimes worsens at night. ${ }^{2,3}$ Additionally, women may report cracking or bleeding of the vulvar skin and perianal area. Rarely is lichen sclerosus asymptomatic and found on routine examination.

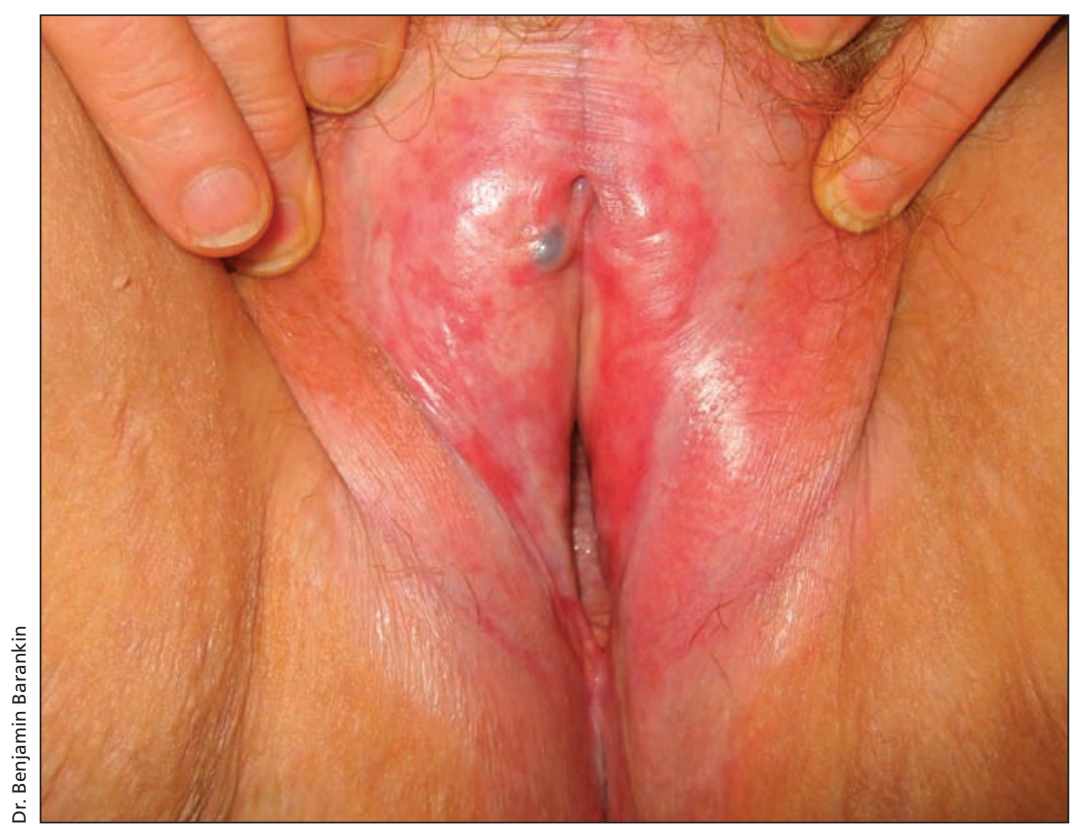

Figure 1: Example of vulvar lichen sclerosus with typical findings, including white plaques, atrophy and narrowing of the introitus.
Physical examination is important for differentiating lichen sclerosus from other causes of vulvar pruritus, such as lichen planus, lichen simplex chronicus, atrophic vaginitis, irritant contact dermatitis, eczema, psoriasis, vulvovaginal candidiasis and skin cancers (e.g., vulvar intraepithelial neoplasia and extramammary Paget disease). ${ }^{1}$ Although findings on examination may vary, the characteristic appearance of lichen sclerosus is ivory white plaques and atrophic skin along the labia with progression to scarring, narrowing of the introitus and ecchymosis or hemorrhage from scratching (Figure 1). Affected areas can look silvery, shiny or crinkly in texture. ${ }^{4}$ The distribution of skin changes may involve the perianal skin, and scarring can cause fusion of the labia minora to the labia majora and flattening of the clitoral hood, which may result in immobility. ${ }^{3}$

\section{Should a biopsy be performed?}

The diagnosis of lichen sclerosus is primarily made by clinical assessment; however, vulvar biopsy is often recommended for histologic confirmation. .,2, $^{2}$ Biopsy is also helpful to exclude precancerous and cancerous lesions, because patients with lichen sclerosus are at increased risk of vulvar cancer. ${ }^{1,5}$ Biopsy with a full-thickness punch technique at the margin of the lesion is considered optimal.

\section{What is the first-line treatment?}

Treatment for lichen sclerosus should begin with a topical steroid such as potent mometasone furoate, $0.05 \%$, or ultrapotent clobetasol propionate, $0.05 \%$. Calcineurin inhibitors, such as $1 \%$ pimecrolimus cream, are considered second-line treatment. ${ }^{4}$ Although dosing recommendations may vary, the dosage most often reported is daily application of clobetasol ointment to the affected area for one month, followed by four weeks of applications every other day, and thereafter twice weekly applications to minimize flare-ups. ${ }^{1,2,4-6}$ Patients should be instructed to use a minimal amount of steroid with each application.

There is no evidence to support the use of topical treatment of genital lichen sclerosus with androgens, progesterone or antibiotics. ${ }^{4,5}$ 


\section{Are nonpharmacologic treatments effective?}

Patients should be educated about symptomatic relief of dyspareunia with vaginal lubricants and about behavioural modifications to reduce scratching. Lichen sclerosus is not routinely treated with surgery unless there is cancer, severe dyspareunia or clitoral phimosis. ${ }^{1,2,5}$ Anecdotal reports have indicated that photodynamic therapy may be effective. ${ }^{4}$

\section{What should this patient be told about the prognosis?}

Patients with lichen sclerosus should be told that this disorder is a chronic condition that can be managed with therapy. Scarring is irreversible, and thus one goal of treatment should be to prevent further progression of disease and further scarring. Because of the $2 \%-5 \%$ increased risk of developing squamous cell carcinoma, patients should be monitored every 6-12 months. ${ }^{1,2,5}$ There may be an additional association with autoimmune disorders such as alopecia areata, vitiligo, thyroid disease and pernicious anemia. Affected patients should be screened for these additional disorders. ${ }^{1}$

\section{The case revisited}

With a presentation of chronic vulvar itching in a postmenopausal woman, a diagnosis of lichen sclerosus should be considered. A biopsy was performed, and a course of high-potency topical steroids prescribed. The patient was monitored closely for the resolution of symptoms or progression in the appearance of lesions.

\section{References}

1. O'Connell TX, Nathan L, Satmary WA, et al. Non-neoplastic epithelial disorders of the vulva. Am Fam Physician 2008;77:321-6.

2. Murphy R. Lichen sclerosus. Dermatol Clin 2010;28:707-15.

3. Thorstensen KA, Birenbaum DL. Recognition and management of vulvar dermatologic conditions: lichen sclerosus, lichen planus and lichen simplex chronicus. J Midwifery Womens Health 2012;57:260-75

4. Chi CC, Kirtschig G, Baldo M, et al. Systematic review and metaanalysis of randomized controlled trials on topical interventions for genital lichen sclerosus. J Am Acad Dermatol 2012;67:305-12.

5. Sadowska-Przytocka A, Danczak-Pazdrowska A, Szewczyk A, et al. Treatment of genital lichen sclerosus in woman - review. Ginekol Pol 2011;83:458-61.

6. Minghetti S, Borghi A, Corazza M. Proactive maintenance therapy with topical corticosteroid for vulvar lichen sclerosus: preliminary results of a randomized study. Br J Dermatol 2013;168:1316-24.

Affiliation: Family Medicine, Kaiser Permanente, Vallejo, Calif.

Contributors: Both authors contributed equally to all aspects of this paper and approved the final version submitted for publication.

Acknowledgement: The authors would like to thank Dr. Benjamin Barankin for providing a representative image of vulvar lichen sclerosus.

Decisions is a series that focuses on practical evidence-based approaches to common presentations in primary care. The articles address key decisions that a clinician may encounter during initial assessment. The information presented can usually be covered in a typical primary care appointment. Articles should be no longer than 650 words, may include one box, figure or table and should begin with a very brief description (75 words or less) of the clinical situation. The decisions addressed should be presented in the form of questions. A box providing helpful resources for the patient or physician is encouraged.

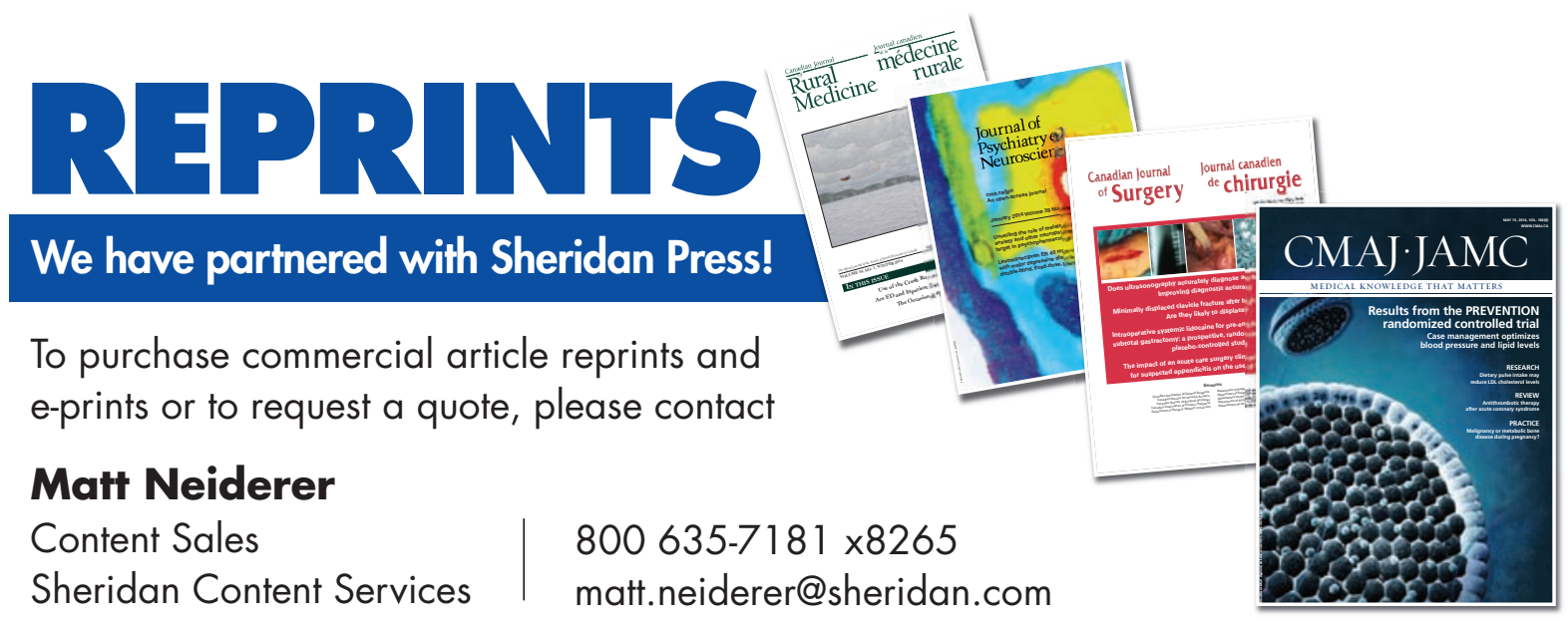

